DOI:

10.1038/nrg2246

\title{
The dawn of recombinant DNA
}

The ability to make recombinant DNA molecules is the cornerstone of modern molecular biology. Yet 40 years ago, it was hardly a conceivable accomplishment.

In the 1960s, biologists had realized that DNA recombination happens in the cell - for example, when breaks caused by ultraviolet irradiation are repaired - and the search for an enzyme that could join DNA molecules was on. The breakthrough came at the beginning of 1967, when Martin Gellert at the National Institutes of Health showed that Escherichia coli extracts could convert $\lambda$ phage DNA 'hydrogenbonded circles' into a covalently circular form. Within 6 months, Gellert and three other groups independently purified the enzymatic activity, which formed phosphodiester bonds between DNA ends held by hydrogen-bond pairing in a double-stranded configuration.

DNA ligase, which was the first ingredient for making recombinant DNA, was then at hand, but other ingredients, like restriction enzymes, (see Milestone 4) remained to be discovered. Another key concept was the use of plasmids as vectors for shuttling DNA into bacteria. Stanley Cohen, who was studying the role of plasmids in bacterial resistance to antibiotics at Stanford University, first worked out a 'transformation' method to make bacteria take up purified plasmid DNA.

Then, in 1973, Cohen and his Stanford colleague Annie Chang, in collaboration with Herbert Boyer and Robert Helling at the University of California in San Francisco, reported the first in vitro construction of a bacterial plasmid. Using the restriction enzyme EcoRI, they generated

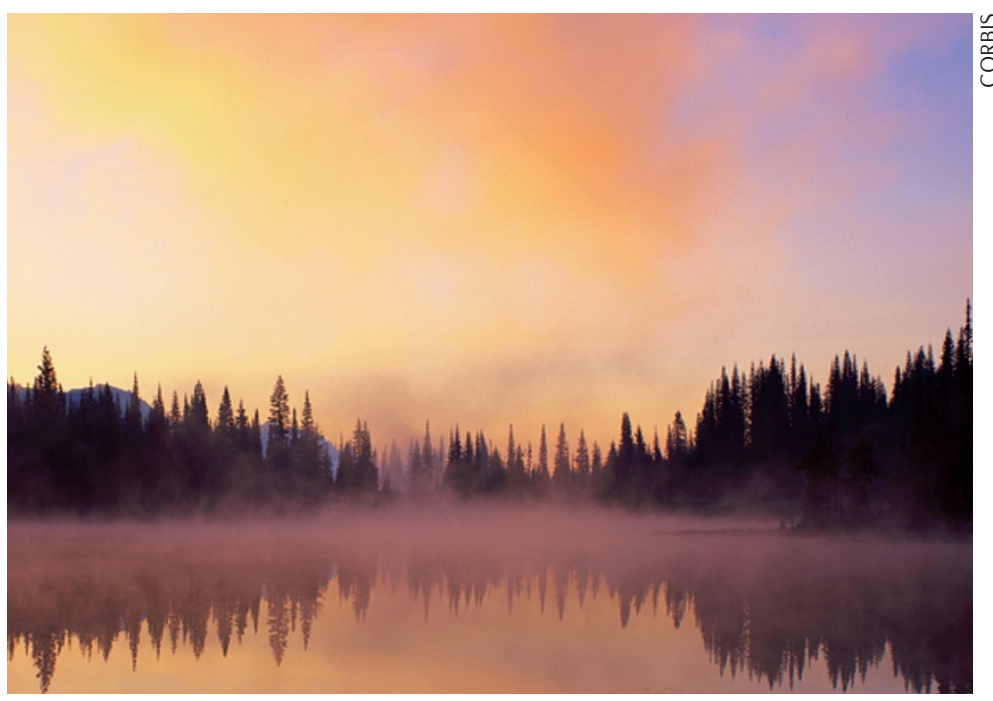

fragments from two plasmids (each conferring resistance to one antibiotic), joined them using DNA ligase and applied the mixture to transform E. coli. As they had hoped, a fraction of the transformed bacteria became resistant to both antibiotics while carrying a single hybrid plasmid. Not only had they demonstrated that bacterial plasmids constructed in vitro were functional in bacteria, but they had also described the first plasmid vector.

Meanwhile, Paul Berg had devised a similar experiment to transfer foreign DNA into mammalian cells, using the tumour virus SV40 as a vector. In 1972, he made a hybrid molecule in vitro by inserting $\lambda$ phage sequences into SV40. These reports immediately raised concerns, as E. coli, which is a natural habitant of the human gut, could now carry hybrid DNA molecules containing SV40 oncogenes or other potentially harmful sequences. These fears led the community to a self-imposed moratorium on recombinant DNA experiments. However, the foundation had been laid and progress soon resumed.

Veronique Kiermer, Chief Editor, Nature Methods

ORIGINAL RESEARCH PAPERS Gellert, M Formation of covalent circles of $\lambda$ DNA by $E$. coli extracts. Proc. Natl Acad. Sci. USA 57, 148-155 (1967) |Cohen, S. N., Chang, A. C. Y. \& Hsu, L. Nonchromosomal antibiotic resistance in bacteria: genetic transformation of $E$. coli by R-factor DNA. Proc. Natl Acad. Sci. USA 69, 2110-2114 (1972)| Cohen, S. N., Chang, A. C. Y., Boyer, H. W. \& Helling, R. B. Construction of biologically functional bacterial plasmids in vitro. Proc. Natl Acad. Sci. USA 70, 3240-3244 (1973)| Jackson, D. A., Symons, R. H. \& Berg, P. Biochemical method for inserting new genetic information into DNA of simian virus 40. Proc. Natl Acad. Sci. USA 69, 2904-2909 (1972)

FURTHER READING Gefter, M. L., Becker, A. \& Hurwitz J. The enzymatic repair of DNA, I. Formation of circular $\lambda$ DNA. Proc. Natl Acad. Sci. USA 58, 240-247 (1967)|Olivera, B. M. \& Lehman, I. R. Linkage of polynucleotides through phosphodiester bonds by an enzyme from E. coli. Proc. Natl Acad. Sci. USA 57, 1426-1433 (1967)| Weiss, B. \& Richardson, C. C. Enzymatic breakage and joining of deoxyribonucelic acid. Proc. Natl Acad. Sci. USA 57, 1021-1028 (1967)|

Zimmerman, S. B., Little, J. W., Oshinsky, C. K. \& Gellert, M. Enzymatic joining of DNA strands: a novel reaction of diphosphopyridine nucleotide. Proc. Natl Acad. Sci. USA 57, 1841-1848 (1967) 